\title{
M otivos e prevalência de insatisfação com a imagem corporal em adolescentes
}

\author{
Reasons and prevalence of body image dissatisfaction in adolescents
}

Edio Luiz Petroski ${ }^{1}$

Andreia Pelegrini ${ }^{1}$

M aria Fátima Glaner ${ }^{2}$
${ }^{1} N$ úcleo de Pesquisa em Cineantropometriae Desempenho Humano, Programa de Pós-

Graduação em Educação Física, Centro de Desportos, UniversidadeFederal de Santa Catarina. Rua Pascoal Simone 358, Coqueiros. 88080-350 Florianopolis SC. petroski@cds.ufsc.br ${ }^{2}$ D epartamento deEducação Física, Centro de Educação Física e Desportos, Universidade Estadual de Londrina
Abstract 0 bjective: To investigate the prevalence of body image (BI) dissatisfaction in adolescents. The study enrolled 641 adolescents aged 11 to 17 from the town of Saudades, Santa Catarina, Brazil. The prevalence of $\mathrm{BI}$ dissatisfaction was $60.4 \%$ ( males $=54.5 \%$, females $=65.7 \% ; p<0.05$ ). Boys were more likely to wish to increase the size of their body silhouette $(26.4 \%)$ while girls wished to reduce theirs (52.4\%). Adolescents from urban areas manifested greater prevalence of $\mathrm{BI}$ dissatisfaction and were more likely to want to reduce their body silhouette. The reasons given by these adolescents for why they were dissatisfied with their body images were similar for both sexes $(p=$ 0.156). When analyzed by neighborhood the urban adolescents said that their dissatisfaction was esthetic, whereas the rural adolescents said it was based on self-esteem. An association was detected between perceived $\mathrm{BI}$ and the reasons for dissatisfaction. Conclusions: Esthetics, self-esteem and health were the most common reasons for $\mathrm{BI}$ dissatisfaction among these adolescents. M ore than half of them were dissatiffied with their body silhouettes. Therefore, there is a need for interventions by health services and professionals in this age range in order to avoid possible future problems with eating disorders (anorexia, bulimia and vigorexia).

Key words Esthetics, Body image, Self esteem
Resumo Objetivo: Verificar os motivose a prevalência de insatisfação com a imagem corporal (IC) em adolescentes. M étodos: Participaram do estudo 641 adolescentes de 11 a 17 anos. A imagem corporal foi autoavaliada através da escala de nove siIhuetas corporais. Resultados: A prevalência de insatisfação com a IC foi de 60,4\% (masculino = $54,5 \%$, feminino $=65,7 \% ; p<0,05)$. Os rapazes $(26,4 \%)$ apresentaram maior desejo em aumentar o tamanho da silhueta corporal, enquanto as moças $(52,4 \%)$ desejavam diminuir. Prevalência mais elevada de insatisfação com a IC foi encontrada nos adolescentes urbanos. Os motivos indicados pelos adolescentes para a insatisfação foram similares entre os sexos $(p=0,156)$. Quando ao domićlio, a estética foi o motivo indicado pelos adolescentes urbanos e a autoestima pelos adolescentes rurais. Foi encontrada associação entre a percepção da imagem corporal e os motivos de insatiffação. Conclusões: A estética, a autoestima ea saúde são os motivos que mais influenciam a insatisfação com a IC. M ais da metade dosadolescentes está insatiffeita com sua silhueta corporal. N este sentido, interven ções por profissionais eserviços de saúde são necessáriasnessa fase da vida, para prevenir possíveis problemas futuros de distúrbios alimentares (anorexia, bulimia e vigorexia).

Palavras-chave Estética, Imagem corporal, Autoestima 
Introdução

A imagem corporal pode ser conceituada como uma construção multidimensional ${ }^{1}$, que representa como os indivíduos pensam, sentem e se comportam a respeito de seus atributos físicos². Ela pode ser vista como a relação entre o corpo de uma pessoa e os processos cognitivos como crenças, valores e atitudes individuais.

A adolescência é caracterizada por transformações biológicas, físicas, psicológicas e sociais s $^{3}$. Atrelado a esse contexto, pesquisas têm revelado elevada prevalência de insatisfação com a imagem corporal em adolescentes, sendo mais acentuada no sexo feminino ${ }^{4-7}$.

As sociedades contemporâneas, principalmente as ocidentais, vêm apresentando uma preocupação excessiva com os padrões de beleza, nas quais há uma verdadeira "divinização" do corpo belo ${ }^{8}$, além de uma busca incessante pela magreza exagerada9. Isto tem contribuído para o aumento da insatisfação com a imagem corporal, acometendo negativamente al guns aspectos da vida dos indivíduos, principalmente no que tange ao comportamento alimentar, psicossocial, físico e cognitivo e à autoestima ${ }^{9,10}$. Com 0 objetivo de alcançar uma efetiva satisfação com a imagem corporal, correspondente aos ideais estéticos da "cultura", é cada vez mais evidente queas pessoas estão recorren do a dietas, ao exercício físico exagerado, ao uso de diuréticos, laxantes, entreoutros recursos. Consequentemente, surgem transtornos alimentares, como a anorexia e a bulimia nervosa ${ }^{11}$.

Percebe-se uma gama de estudos $s^{4,7,12-15}$ sobre a percepção da imagem corporal em adolescentes, os quais investigaram a prevalência de insatisfação corporal e a associação com indicadores antropométricos. Todavia, não foram encontrados estudos que verificassem os motivos que levam à insatisfação corporal. Para tanto, o objetivo do presente estudo foi verificar os motivose a prevalência de insati sfação com a imagem corporal em adolescentes.

\section{M étodo}

O estudo sobre motivos e prevalência de insatisfação com a imagem corporal em adolescentes foi desenvolvido a partir de um estudo transversal denominado "Interação entre variáveis que podem influenciar no acúmulo de gordura corporal eno perfil lipídico de pais efilhos", aprovado por um comitê de ética institucional, efoi rea- lizado em uma amostra representativa de adolescentes, domiciliados nas áreas urbana e rural, do município de Saudades (SC), localizado no extremo Oeste do estado de Santa Catarina, re gião Sul do Brasil.

O município de Saudades é predominantemente formado por descendentes de Alemães e também, em menor número, por Russos, I talianos e outros ${ }^{16}$. Caracteriza-se por ser um município de pequeno porte, com estimativa ${ }^{17}$ de 8.880 habitantes, para o ano 2008. 0 seu Índice de Desenvolvimento H umano ${ }^{18}$ é 0,82 , sendo classificado como alto. Em 2008, o Índice de Desenvolvimento da Educação foi o 180 no estado ${ }^{16}$.

A população do estudo foi composta por 1.381 adolescentes, com idade de 10 a 17 anos ${ }^{17}$, matriculados em escolas públicas. Não há escolas privadas em Saudades. Foi selecionada, intencionalmente, a única escola queatendealunos da 5 a série do ensino fundamental até o último ano do ensino médio, localizada na sede do município. Outra, localizada na área rural, queatende alunos do ensino fundamental. O salunos, da respectiva faixa etária envolvida no estudo, foram convidados em sala de aula e por meio de uma "carta convite" que levaram aos pais ou responsáveis legais.

Foram calculados vários tamanhos de amostra, pois este estudo faz parte de uma pesquisa mais ampla, com diferentes desfechos em saúde. Para a presente análise, a amostra escolhida foi aquela selecionada para o estudo sobre prevalência insatisfação com a imagem corporal. Adotou-se uma prevalência para o desfecho de $64 \%{ }^{7}$, intervalo de confiança $95 \%$ (IC $95 \%$ ), erro estimado de cinco pontos percentuais, efeito do delineamento de 1,6 eacréscimo de $30 \%$ como possível índice de perda. Assim, estimou-seque seria necessário coletar informações de 586 adolescentes. Em virtude das características do processo de amostragem que envolveu todos os indivíduos pertencentes aos conglomerados (escolas), participaram da amostra 708 adolescentes.

Asinformações da percepção da imagem corporal foram obtidas com a utilização da escala de nove silhuetas corporais proposta por Stunkard et al. ${ }^{19}$. O conjunto de silhuetas era mostrado aos adolescentes, os quais respondiam a duas perguntas: Qual a silhueta que melhor representa a sua aparência corporal atual (real)? Qual é a silhueta corporal que você gostaria de ter (ideal)? Quando a variação entre a silhueta real ea ideal era igual a zero, os adolescentes eram classificados como satisfeitos; e se diferente de zero, insatisfeitos. Caso a diferença fosse positi- 
va (real - ideal), era uma insatisfação pelo desejo de reduzir a silhueta e, quando negativa, uma insatisfação pelo desejo de aumentar. Em caso de insatisfação, os adolescentes responderam a mais uma pergunta: Qual o principal motivo da insatisfação? As respostas obtidas foram categorizadas nos seguintes motivos: estética, saúde, meIhora nas atividades diárias, autoestima, opinião familiar e desejo de ser fortefisicamente. Devido ao baixo número de adolescentes que responderam melhora nas atividades diárias ( $n=12$ ), opinião familiar ( $n=3$ ) e desejo de ser forte fisicamente $(n=4)$, estes foram categorizados em outros motivos.

N as comparações entre duas proporções, foi aplicado o teste de significância para diferenças entre proporções. Para verificar as possíveis diferenças de frequência entre as variáveis, utilizou-se o teste qui-quadrado. 0 nível de significância adotado foi $p<0,05$.

\section{Resultados}

Foram excluídos da amostra os adolescentes com idade superior a 17 anos $(n=48)$. Considerouse como perda amostral os adolescentes que não responderam os questionários sobre percepção da imagem corporal $(n=23)$ e/ou os motivos de insatisfação corporal $(n=2)$. D esta forma, a amostra final foi composta por 641 adolescentes
(303 rapazes e 338 moças), sendo 368 domiciliados na área urbana e 273 na área rural, com idades de 11 a 17 anos.

A média de idade decimal dos adolescentes envolvidos no estudo foi de 14,3 anos $( \pm 1,9)$. A prevalência de insatisfação com a imagem corporal foi de $60,4 \%$. A Figura 1 apresenta a distribuição dos adolescentes de acordo com a percepção da imagem corporal. As moças (65,7\%) apresentaram maior insatisfação corporal que os rapazes (54,5\%; $p<0,05$ - Figura 1A). Quando a insatisfação foi dicotomizada em deseja aumentar e deseja reduzir o tamanho da silhueta corporal (Figura 1B), diferenças $(p<0,05)$ entre os sexos foram verificadas. Enquanto os rapazes desejavam aumentar o tamanho da silhueta corporal $(26,4 \%)$, as moças desejavam reduzir $(52,4 \%)$.

A Figura 2 apresenta os resultados referentes à percepção da imagem corporal por área de domicílio. Verificou-se maior prevalência ( $p<$ 0,05 ) de insatisfação com a imagem corporal nos adolescentes domiciliados na área urbana (65,2\%) , em relação àqueles daárea rural $(53,5 \%$ - Figura 2A). Quando a percepção da imagem corporal foi dicotomizada (deseja aumentar e deseja reduzir - Figura 2B), observou-se que os adolescentes da área urbana (44,7\%) apresentaram maior desejo em reduzir o tamanho da siIhueta corporal que os da área rural $(35,9 \%)$.

Os motivos indicados pelos adolescentes para a insatisfação com a imagem corporal foram si-

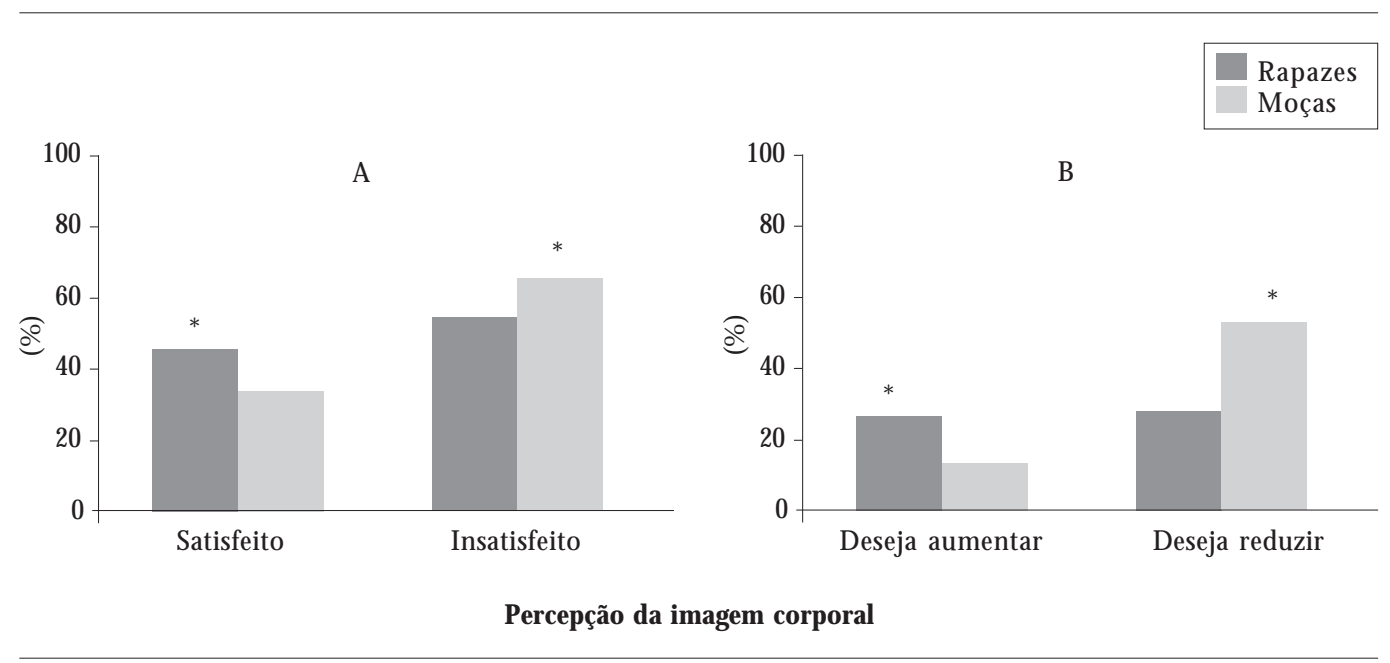

Figura 1. Distribuição dos adolescentes de acordo com a percepção da imagem corporal e sexo. Saudades, Santa Catarina.

$A=I$ magem corporal categorizada de forma dicotômica $\left(\chi^{2}=8,415 ; p<0,05\right) ; B=0$ grupo insatisfeito foi dicotomizado em duas categorias: deseja aumentar e deseja reduzir $\left({ }^{*} \chi^{2}=42,226 ; p<0,001\right)$. 
milares entre os sexos $(p=0,156)$. A pesar de não ter sido encontrada diferença, percebe-se que a estética ( masculino $=45,9 \%$, feminino $=52,4 \%$ ) e a autoestima ( masculino $=45,9 \%$, feminino $=$ $39,9 \%$ ) são os motivos (Figura 3A) que mais influenciam na distorção da imagem corporal em adolescentes. Em relação à área de domicílio (Figura 3B), observou-se que a estética $(53,7 \%)$ influencia a distor ção da imagem corporal nos adolescentes urbanos, enquanto a autoestima $(50,9 \%)$ aparece como o motivo que mais distorce a imagem corporal nos adolescentes rurais.
Foi encontrada associação entre a percepção da imagem corporal e motivos de insatisfação. Verificou-se que a estética (96,8\%), a saúde $(95,5 \%)$ e outros ( $100 \%)$ são os motivos que mais influenciam a insatisfação com a imagem corporal em adolescentes (Figura 4A). Quando a insatisfação foi dicotomizada em "deseja aumentar" e "deseja reduzir" o tamanho da silhueta corporal, observou-se que os principais motivos indicados pelos adolescentes que desejam aumentar o tamanho da silhueta corporal foram: meIhora nas atividades diárias, opinião familiar e o
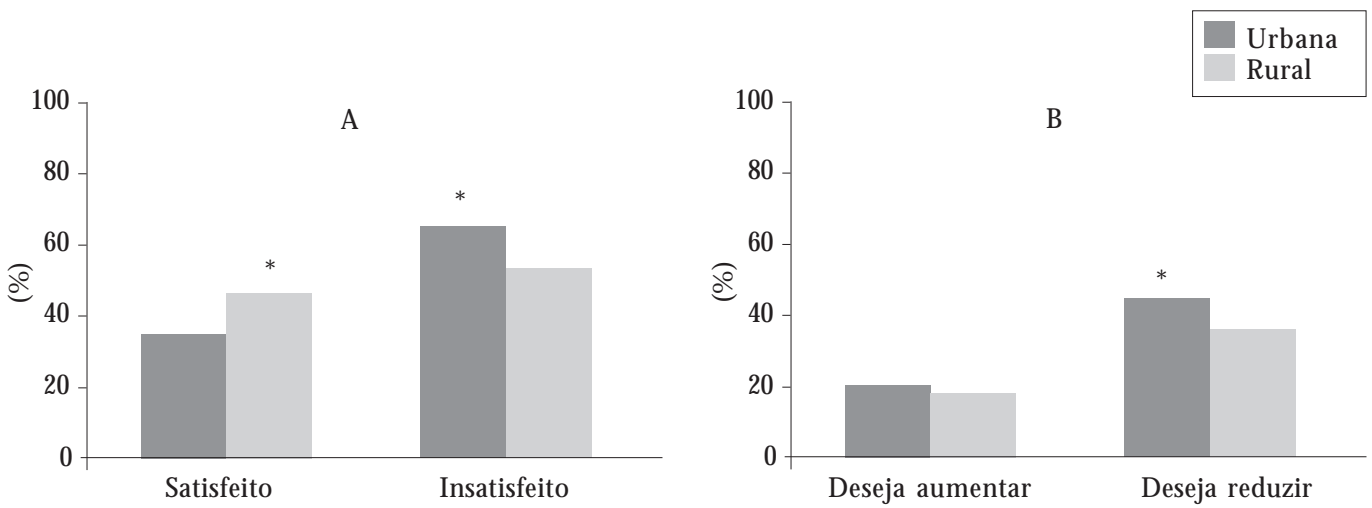

Percepção da imagem corporal

Figura 2. Distribuição dos adolescentes de acordo com a percepção da imagem corporal por área de domicílio. Saudades, Santa Catarina.

$A=I$ magem corporal categorizada de forma dicotômica $\left(\chi^{2}=8,962 ; p<0,05\right) ; B=0$ grupo insatisfeito foi dicotomizado em duas categorias: deseja aumentar e deseja reduzir $\left({ }^{*} \chi^{2}=9,036 ; p<0,05\right)$

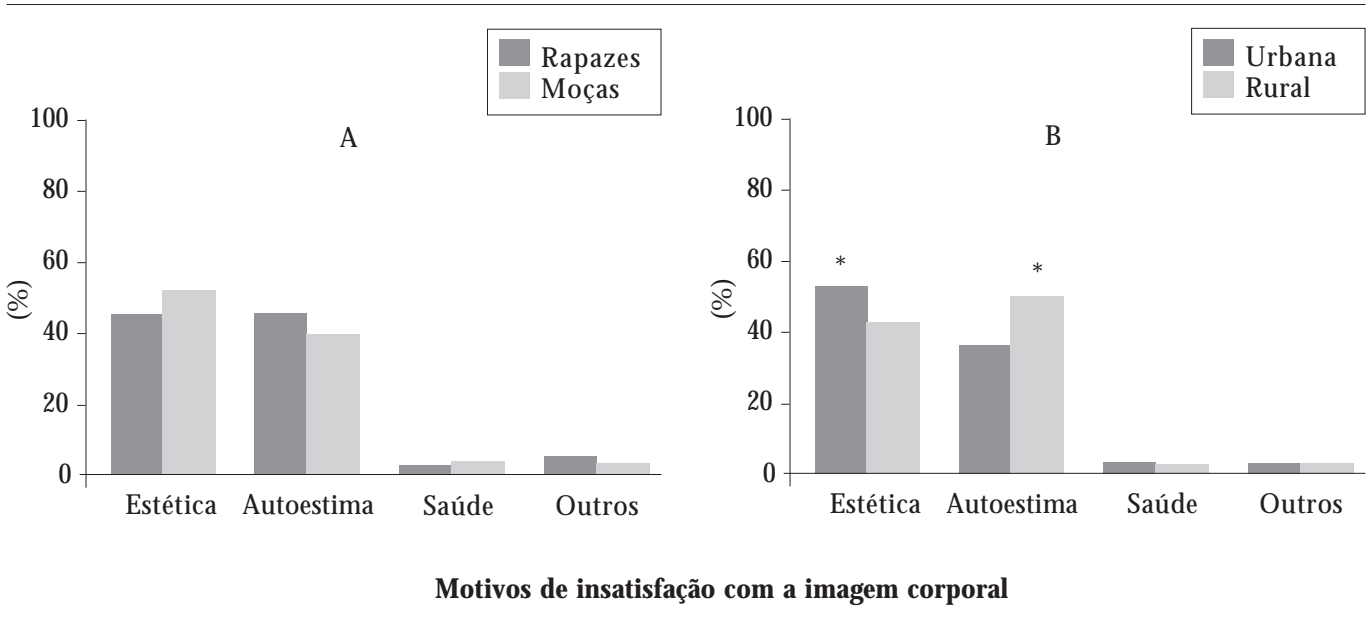

Figura 3. M otivos para insatisfação com a imagem corporal em adolescentes, por sexo - $\mathrm{A}\left(\chi^{2}=5,231\right.$; $\mathrm{p}=0,156)$ e área de domicílio - B $\left(\chi^{2}=12,943 ; p<0,05\right)$. Saudades, Santa Catarina. 
desejo de ser forte fisicamente $(44,8 \%)$ e estética (32,0\%). Por outro lado, os motivos apontados pelos adolescentes que desejam reduzir o tamanho da silhueta corporal foram, na ordem decrescente, autoestima $(88,7 \%)$, saúde $(86,4 \%)$, estética $(64,9 \%)$ e outros $(55,2 \%)$ (Figura 4B).

\section{Discussão}

A prevalência de insatisfação com a imagem corporal foi de $60,4 \%$. Prevalências similares aos do presente estudo foram encontradas em dois municípios do Rio Grande do Sul7; superiores em Porto Alegre (RS) ${ }^{6}$ e Três de Maio $(R S)^{4}$ e inferiores em Florianópolis (SC ${ }^{20}$. Em pesquisa realizada em três municípios do O este catarinense, inclusive Saudades (SC), eum do N orte Gaúcho ${ }^{15}$, os resultados revelaram que $63,4 \%$ dos adolescentes domiciliados na área rural eurbana estavam insatisfeitos com a imagem corporal.

Observou-se maior prevalência de insatisfação com a imagem corporal nas moças (65,7\%), quando comparada aos rapazes (54,5\%). Possivelmente, essa maior insatisfação com a imagem corporal entre as adolescentes decorre da pressão exercida pela mídia e pela sociedade, que impõe, cada vez mais, padrões de beleza caracterizados pela magreza excessiva ${ }^{21}$. Investigações demonstram queem adolescentes, principalmente do sexo feminino, a insatisfação com a imagem corporal está diretamenterelacionada ao desenvolvimento de problemas e distúrbios alimentares ${ }^{22,23}$. Contrastando com estes achados, recente pesquisa conduzida em adolescentes domiciliados na área urbana e rural, revelou semelhança na insatisfação com a imagem corporal entre os sexos ${ }^{15}$.

Enquanto os rapazes desejam aumentar $(26,4 \%)$, as moças desejam diminuir $(52,4 \%) 0$ tamanho da silhueta corporal. Esses resultados corroboram os estudos nacionais $s^{6,7,15}$. Estudo realizado em Três de Maio (RS), somente com moças ( $10-17$ anos), revelou que $71,7 \%$ e 13,3\% das adolescentes desejavam reduzir e aumentar a silhueta corporal, respectivamente ${ }^{4}$. Recente pesquisa conduzida em crianças e adolescentes da cidade de Florianópolis (SC) revelou que as moças desejam corpos mais magros e os rapazes desejam corpos mais fortes ${ }^{14}$.

M aior prevalência de insatisfação com a imagem corporal foi verificada nos adolescentes urbanos quando comparados aos rurais. Além disso, os indivíduos domiciliados na área urbana apresentaram maior desejo em reduzir o tamanho da silhueta corporal. Resultados semelhantes foram encontrados em pesquisa realizada com escolares de dois municípios do Rio Grande do Sul ${ }^{7}$. Os achados encontrados por Petroski et al. ${ }^{15}$ revelaram semel hança na prevalência de insatisfação com a imagem corporal entre as áreas de domicílio (urbana $=62,8 \%$, rural $=64,2 \%$ ). De modo geral, o descontentamento com a imagem corporal está presente mesmo nos adolescentes das áreas rurais.
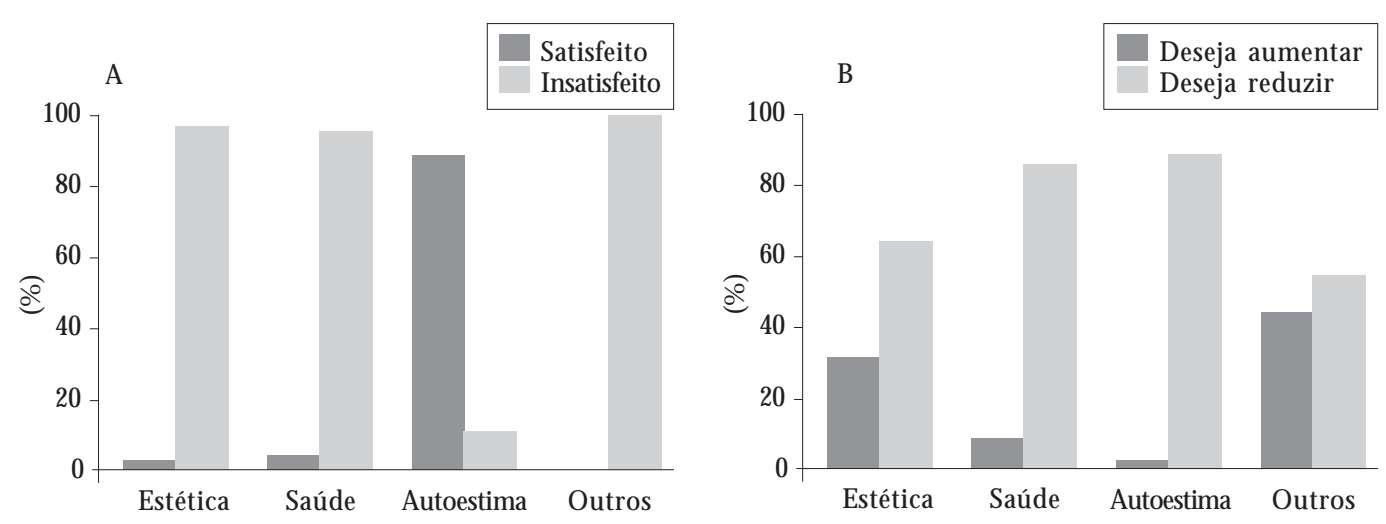

Motivos de insatisfação com a imagem corporal

Figura 4. Percepção da imagem corporal categorizada por motivos em adolescentes. Saudades, Santa Catarina. 
Foi encontrada associação entre a percepção da imagem corporal com os motivos. A estética e a saúde foram os motivos que mais influenciaram na insatisfação com a imagem corporal em adolescentes. Ao ser verificada a insatisfação com a imagem corporal em adolescentes de Florianópolis (SC) observou-se que 5,6\% das adolescentes indicaram motivos de ordem estética para a insatisfação com a imagem corporal ${ }^{20}$. Em pesquisa de natureza quanti-qualitativa conduzida em adolescentes de Vitória (ES), observou-se a importância dada à estética, como principal motivo pelo desejo de mudanças ${ }^{24}$.

Os dados do presente estudo apontaram que enquanto nos adolescentes urbanos a estética foi o maior motivo de insatisfação com a imagem corporal, nos rurais, a autoestima apareceu como o motivo de maior influência na distorção da imagem corporal. Assis et al. ${ }^{25}$ ao investigarem 0 autoconceito e a autoestima de estudantes de São Gonçalo (RJ), revelaram que os adolescentes estão satisfeitos com o seu corpo, pois revelaram autoconceito positivo e autoestima elevada. Por um lado, percebe-se que os adolescentes com autoestima elevada acreditam na sua competência, valor e em si mesmos; por outro lado, aqueles com baixa autoestima sentem-se inferiorizados, desval orizam-se e sentem-se inseguros.

A preocupação com o corpo, no quese refereà estética, é popularizada e banalizada através dos meios de comunicação. A globalização também traz model os de outros países e regiões, quemuitas vezes não condizem com as características morfológicas de determinada etnia egrupo deadolescen- tes $^{26}$. Além disso, a imagem corporal exerce influência na autoestima dos adolescentes, sendo que eles colocam mais importância em sua aparência queosadultos. Estasobservações demonstram que a aparência éum aspecto altamentesal ientenaformação da identidade dos adolescentes ${ }^{27}$.

As principais limitações desseestudo são: de lineamento transversal, ou seja, estima as relações entre as variáveis em apenas um único momento e não permite identificar as relações de causa e efeito; método de avaliação da imagem corporal, que provém informação limitada por ser bidimensional, o que não permite arepresentação do indivíduo como um todo, distribuição da massa de gordura subcutânea e aspectos antropométricos importantes na formação da imagem corporal ${ }^{28}$. Entretanto, destaca-se que esteé o primeiro estudo brasileiro a investigar os motivos da prevalência de insatisfação com a imagem corporal em adolescentes.

\section{Conclusão}

De acordo com os achados do presente estudo, conclui-se que a estética, a autoestima e a saúde são os motivos que mais influenciam a insatisfação com a imagem corporal em adolescentes. $M$ ais da metade dos adolescentes está insatisfeita com sua silhueta corporal. Neste sentido, intervenções por profissionais e serviços de saúde são necessárias nessa fase da vida, evitando possíveis problemas futuros de distúrbios alimentares (anorexia, bulimia e vigorexia).

\section{Colaboradores}

EL Petroski, A Pelegrini e M F Glaner participaram igualmente de todas as etapas de elaboração do artigo. 


\section{Referências}

1. Thompson JK. Body image disturbance: assessment and treatment. New York: Pergamon; 1990.

2. Muth JL, Cash TF. Body-image attitudes: what difference does gender make? J Appl Soc Psychol 1997; 27(16):1438-1452.

3. Campagna VN, Souza ASL. Corpo e imagem corporal no início da adolescência feminina. Boletim de Psicologia 2006; 56(124):9-35.

4. Corseuil MW, Pelegrini A, Beck CC, Petroski EL. Prevalência de insatisfação com a imagem corporal e sua associação com a inadequação nutricional em adolescentes. Rev Educ Fís (UEM) 2009; 20(1): 25-31.

5. Graup S, Pereira EF, Lopes AS, Araújo VC, Legnani RFS, Borgatto AF. Associação entre a percepção da imagem corporal e indicadores antropométricos de escolares. Rev Bras Educ Fis Esp 2008; 22(2):129-138.

6. Pinheiro AP, Giugliani ERJ. Body dissatisfaction in Brazilian schoolchildren: prevalence and associated factors. Rev Saude Publica 2006; 40(3):489-496.

7. Triches RM, Giugliani ERJ. Insatisfação corporal em escolares de dois municípios da região Sul do Brasil. Rev Nutr 2007; 20(2):119-128.

8. Alves $D$, Pinto $M$, Alves $S$, M ota A, Leirós V. Cultura e imagem corporal. M otricidade 2009; 5(1):1-20.

9. Smolak L. Body image in children and adolescents: where do we go from here? Body I mage 2004; 1(1):1528.

10. Hargreaves DA, Tiggemann M. Idealized media images and adolescent body image: "comparing" boys and girls. Body Image 2004; 1(4):351-361.

11. Morgan C, Vecchiatti I, Negrão A. Etiologia dos transtornos alimentares: Aspectos biológicos, psicológicos e socioculturais. Rev Bras Psiq 2002; 24(Supl.3):18-23.

12. Branco LM, Hilário MOE, Cintra IP. Percepção e satisfação corporal em adolescentes e a relação com seu estado nutricional. Rev Psiq Clín 2006; 33(6):292296

13. Conti MA, Frutuoso MFP, Gambardella AMD. Excesso de peso e insatisfação corporal em adolescentes. Rev Nutr 2005; 18(4):491-497.

14. Pereira EF, Graup S, Lopes AS, Borgatto AF, Daronco LSE. Percepção da imagem corporal de crianças e adolescentes com diferentes níveis socioeconômicos na cidade de Florianópolis, Santa Catarina, Brasil. Rev Bras Saúde M atern Infant 2009; 9(3): 253-262.

15. Petroski EL, Pelegrini A, Glaner M F. Insatisfação corporal em adolescentes rurais e urbanos. M otricidade 2009; 5(4):13-25.

16. Prefeitura M unicipal Saudades. Saudades. [site na Internet] [acessado 2009 mar 11]; Disponível em: http://www.saudades.sc.gov.br.

17. Brasil. M inistério da Educação, Instituto Nacional de Estudos e Pesquisas Educacionais (INEP). Censo Educacional. [página na Internet]. [acessado 2008 nov 10]; Disponível em: http://www.ibge.gov.br/cidadesat/topwindow.html.
18. Programa das Nações Unidas para o Desenvolvimento (PNUD). Ranking do Índice de Desenvolvimento M unicipal dos municípios do Brasil. [site na Internet]. [acessado 2009 Set 18]; Disponível em: http://www.pnud.org.br/atlas/tabelas/index.php

19. Stunkard AJ, Sorenson T, Schlusinger F. Use of the Danish adoption register for the study of obesity and thinness. In: Kety SS, Rowland LP, Sidman RL, Matthysse SW, editors. The genetics of neurological and psychiatric disorders. New York, NY: Raven; 1983. p. 115-120.

20. Petroski EL, Velho NM, De Bem MFL. Idade de menarca e satisfação com o peso corporal. Rev Bras Cineantropom Desempenho Hum 1999; 1(1):30-36.

21. Van den Berg P, Paxton SJ, Keery H, Wall M, Guo J, Neumark-Sztainer D. Body dissatisfaction and body comparison with media images in males and females. Body Image 2007; 4(3):257-268.

22. Alves E, Vasconcelos FAG, Calvo M CM, Neves J. Prevalência de sintomas de anorexia nervosa e insatisfação com a imagem corporal em adolescentes do sexo feminino do M unicípio de Florianópolis, Santa Catarina. Cad Saude Publica 2008; 24(3):503-512.

23. Martins CR, Pelegrini $A, M$ atheus $S C$, Petroski EL. Insatisfação com a imagem corporal e sua relação com estado nutricional, adiposidade corporal e sintomas de anorexia e bulimia em adolescentes. Rev Psiquiatr RS 2010; 32(1):19-23.

24. Braga PD, Molina M CB, Cade NV. Expectativas de adolescentes em relação a mudanças do perfil nutricional. Cien Saude Colet 2007; 12(5):1221-1228.

25. Assis SG, Avanci JQ, Silva CM FP, M alaquias JV, Santos NC, Oliveira RVC. A representação social do ser adolescente: um passo decisivo na promoção da saúde. Cien Saude Colet 2003; 8(3):669-680.

26. Garcia RWD. Reflexos da globalização na cultura alimentar: considerações sobre as mudanças na alimentação urbana. Rev Nutr 2003; 16(4):483-492.

27. Dittmar H, Lloyd B, Dugan S, Halliwell E, Jacobs $\mathrm{N}$, Cramer H. The "Body Beautiful": English Adolescents' Images of Ideal Bodies. Sex Roles 2000; $12(9-10): 55-62$

28. Ricciardelli LA, M CCabe M P, Banfield S. Sociocultural influences on body image and body changes methods. J Adolesc Health 2000; 26(1):3-4.

Apresentado em 17/03/2010

Aprovado em 29/04/2010

Versão final apresentada em 21/06/2010 